NASA/TM-2001-211156

AIAA-2001-3318

\title{
A Self-Diagnostic System for the M6 Accelerometer
}

Patrick M. Flanagan

Cleveland State University, Cleveland, Ohio

John Lekki

Glenn Research Center, Cleveland, Ohio

Prepared for the

37th Joint Propulsion Conference and Exhibit

cosponsored by the AIAA, SAE, AIChE, and ASME

Salt Lake City, Utah, July 8-11, 2001

National Aeronautics and

Space Administration

Glenn Research Center 
Available from

NASA Center for Aerospace Information

National Technical Information Service 7121 Standard Drive

Hanover, MD 21076

5285 Port Royal Road Springfield, VA 22100

Available electronically at http://gltrs.grc.nasa.gov/GLTRS 


\title{
A Self-Diagnostic System for the M6 Accelerometer
}

\author{
Patrick M. Flanagan \\ Cleveland State University \\ Cleveland, Ohio 44115 \\ John Lekki \\ National Aeronautics and Space Administration \\ Glenn Research Center \\ Cleveland, Ohio 44135
}

\begin{abstract}
The design of a Self-Diagnostic (SD) accelerometer system for the Space Shuttle Main Engine is presented. This retrofit system connects diagnostic electronic hardware and software to the current M6 accelerometer system. This paper discusses the general operation of the M6 accelerometer SD system and procedures for developing and evaluating the SD system. Signal processing techniques using M6 accelerometer diagnostic data are explained. Test results include diagnostic data responding to changing ambient temperature, mounting torque and base mounting impedance.

\section{Background}

Self-diagnostic accelerometers are needed when the accuracy of data are mission critical. This is especially important in "smart" aerospace systems where a computer system makes judgments and takes action during flight, based on data from these sensors. Smart systems need real-time information on accelerometer performance including soft failures, to be reliable enough for aerospace applications. Accelerometer malfunctions such as improper or shifting mounting torque levels and non-catastrophic damage can produce poor data. These failures should be identified immediately so that appropriate action can be taken by a
\end{abstract}

smart aerospace system. The SD system has been researched with the goal to improve the real-time performance of smart sensor systems with the reliability that is necessary for implementation in aerospace vehicles.

A SD system makes use of the accelerometer's piezoelectric crystals to measure both acceleration and diagnostic data. This system does not change the structure or the acceleration measurement capabilities of the M6 accelerometer. SD operation uses both forward and reverse piezoelectric effect to simultaneously measure acceleration and detect soft/hard failures. Both acceleration and diagnostic data are collected in real-time.

Traditionally, an accelerometer's low frequency band is reserved for acceleration measurements. To facilitate real-time detection, the SD system uses a separate, higher frequency band. Diagnostic data from this frequency band is collected by means of the reverse piezoelectric effect. Empirical models correlating diagnostic signatures to soft and hard failures are employed to determine the operating condition of the accelerometer. SD functions are developed from these empirical models. The initial design criterion for the M6 SD system is to detect several types of soft failures including when the accelerometer becomes loose from the base mount and changes in temperature. 
Previous studies (ref.1-6) have proposed several signal processing techniques for evaluation of diagnostic data. These techniques include means for identifying an acceptable diagnostic frequency band and analyzing narrow-band as well as broadband data. Speed and accuracy of diagnostics are major issues for this application. Since several diagnostic events can occur simultaneously, the capability of the SD system to separate and distinguish a complete diagnostic record is important.

\section{The M6 Self-Diagnostic Accelerometer System}

The M6 Self-Diagnostic (SD) Accelerometer System continuously monitors for problems. As shown in Figure 1, the M6 SD Accelerometer Control System separates acceleration and diagnostic data in-situ. The Diagnostic Generator provides an electrical signal to drive the M6 crystals. It also measures the resulting diagnostic signature and formats SD data to detect soft and hard failures. The Accelerometer Signal Processor collects the acceleration signal, removes the low frequency characteristics of the Diagnostic Generator's electrical drive, and provides a buffered acceleration signal. The SD Control System furnishes a continuous stream of acceleration data with a $20 \mathrm{kHz}$ bandwidth. This system provides digital diagnostic information with a periodicity based on a discrete cycle time. The diagnostic cycle time is primarily controlled by the signal processing algorithm employed. The initial goal of the M6 SD accelerometer system is to provide diagnostic information once a second.

\section{Developing the SD Functions}

A SD function is a mathematical template used to either compensate diagnostic signatures for environmental conditions, i.e. temperature, or identify soft/hard failures. The frequency band of a SD function includes the primary resonance of the piezoelectric accelerometer, both mounted and free. A simple multi-port model of an accelerometer, as shown in figure 2, illustrates the inter-relationship among the accelerometer's electrical, mechanical and piezoelectric properties when using both the forward and reverse piezoelectric effect. A SD function applies specific temporal and spectral characteristics of the reverse piezoelectric effect that relate a dynamic current source, $\mathrm{I}(\mathrm{s})$, to the sensor's output voltage, $\mathrm{E}_{\mathrm{RI}}(\mathrm{s})$.

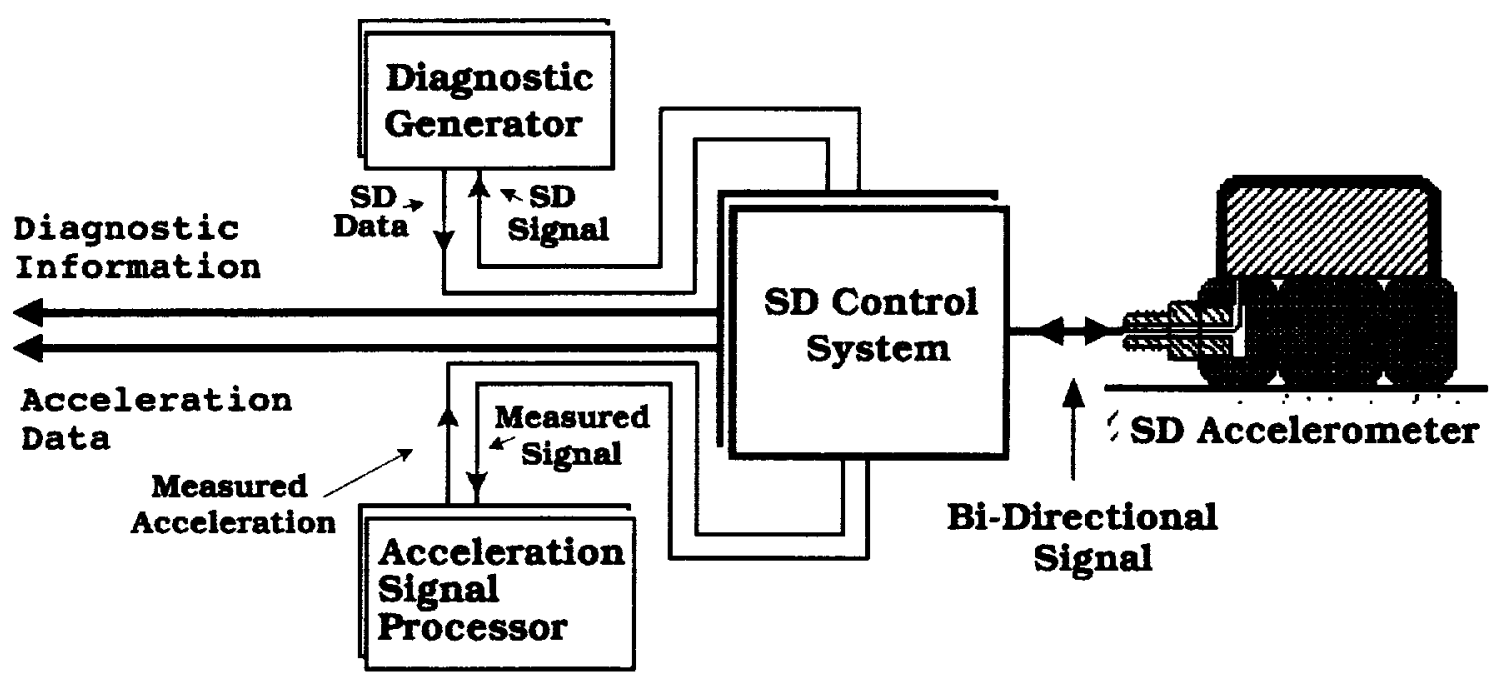

Figure 1: Schematic of the M6 Self-Diagnostic Accelerometer System. 

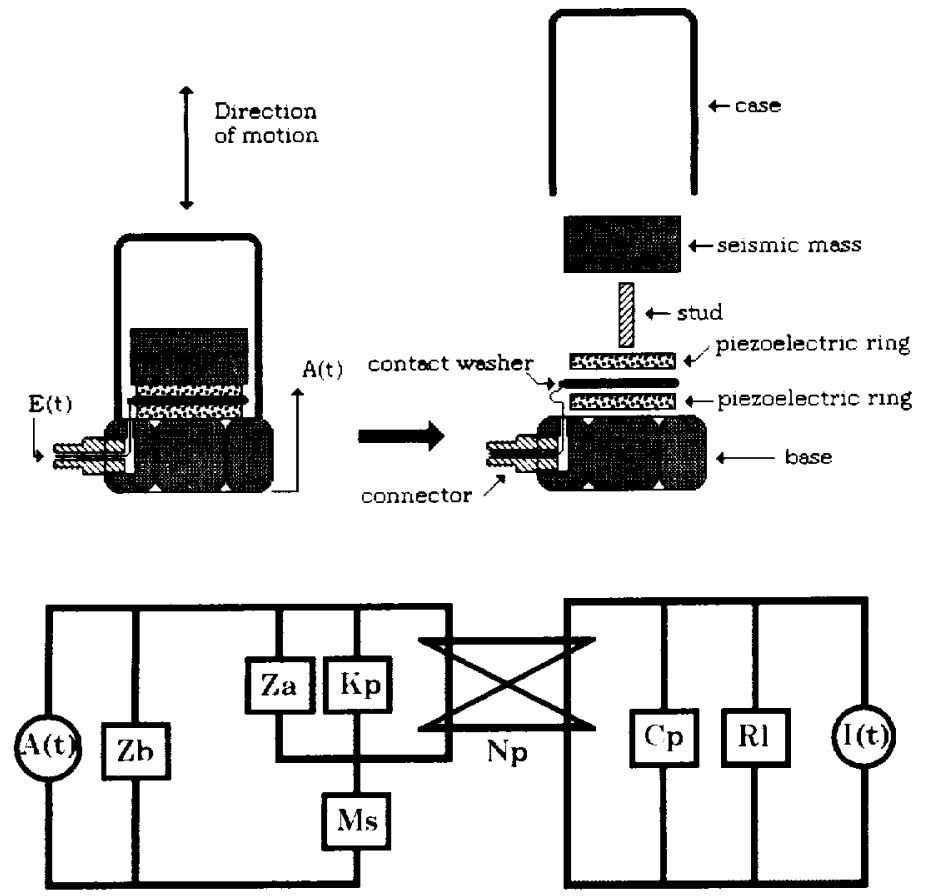

Figure 2; A compression-mode design accelerometer is shown with a simple multi-port model including input ports for both acceleration and a high-frequency current source. Mechanical components considered in this model include base and sensor housing impedance. $Z \mathrm{~b}$. sensor assembly impedance, $\mathrm{Za}$, seismic mass, Ms, and piezoelectric crystal stiffness, Kp. The electrical components include piezoelectric capacitance, $\mathrm{Cp}$ and insulation load resistance, $R_{\mathbf{J}}$. The sensitivity of the accelerometer is the ratio of output voltage across the load resistance to input acceleration. The SD frequency response relates dynamic current to output voltage across the load resistance.

Transfer Function of Forward Piezoelectric Effect

$$
\mathbf{E}_{\mathrm{Rl}}(s)=\frac{[(\mathrm{NpRlMs}) s] \mathbf{A}(s)}{\left[\left(\mathrm{Ms} \mathrm{Np}^{2} \mathrm{RlCp}\right) s^{3}+\left(\mathrm{Za} \mathrm{Np}^{2} \mathrm{RlCp}+\mathrm{Ms} \mathrm{Np}^{2}\right) s^{2}+\left(\mathrm{Za} \mathrm{Np}^{2}+\mathrm{Kp} \mathrm{Np}^{2} \mathrm{RlCp}+\mathrm{Rl}\right) s+\mathrm{Kp} \mathrm{Na}^{2}\right]}
$$

\section{Transfer Function of Reverse Piezoelectric Effect}

$$
\mathbf{E}_{\mathrm{R} 1}(s)=\frac{\mathrm{Np}^{2} \mathrm{Rl}\left[\mathrm{Ms}(\mathrm{Za}+\mathrm{Zb}) s^{2}+(\mathrm{Ms} \mathrm{Kp}+\mathrm{ZaZb}) s+\mathrm{Zb} \mathrm{Kp}\right] \mathbf{I}(s)}{\left[\mathrm{A}_{1} s^{3}+\mathrm{A}_{2} s^{2}+\mathrm{A}_{3} s+\mathrm{A}_{4}\right]}
$$

where:

$$
\begin{aligned}
& \mathrm{A}(\mathrm{s}) \quad=\text { acceleration signal } \\
& \mathrm{Cp} \quad=\text { piezoelectric capacitance } \\
& \mathrm{E}_{\mathrm{RI}}(\mathrm{s}) \text { = voltage output } \\
& \mathrm{I}(\mathrm{s}) \quad=\text { dynamic current source } \\
& \mathrm{Kp} \quad=\text { piezoelectric crystal stiffness } \\
& \text { Ms = seismic mass } \\
& \mathrm{Np} \quad=\text { piezoelectric sensitivity } \\
& \text { RI = load resistance } \\
& s \quad=\text { Laplace transform } \\
& \mathrm{Za} \quad=\text { assembly impedance } \\
& \mathrm{Zb} \quad=\text { base impedance } \\
& A_{1}=(Z a+Z b) M s p^{2} R l C p \\
& A_{2}=\left(Z b Z a p^{2} R l C p+M s Z a p^{2}+M s N p^{2} Z b+M s R l+M s K p N p^{2} R l C p\right) \\
& A_{3}=\left(Z b Z a p^{2}+Z b R l+Z b K p p^{2} R l C p+M s K p ~ N p^{2}\right) \\
& \mathrm{A}_{4}=\mathrm{Zb} \mathrm{Kp} \mathrm{Np}{ }^{2}
\end{aligned}
$$


The two transfer functions show the connection between forward and reverse piezoelectric effect. The transfer function for the forward piezoelectric effect relates dynamic acceleration, $A(s)$, to output sensor voltage, $\mathrm{E}_{\mathrm{Rl}}(\mathrm{s})$. In this case, the current source, I(s), approaches zero within this low frequency measurement band. Only the cyclic beat of the dynamic current source appears at a discrete low frequency and can be removed from the measured acceleration data. The transfer function for the reverse piezoelectric effect relates the dynamic current source, I(s), to output sensor voltage, $E_{\mathrm{Rl}}(\mathrm{s})$. Acceleration in the self-diagnostic frequency band may add noise to the measured voltage, $\mathrm{E}_{\mathrm{RI}}(\mathrm{s})$. If necessary, increasing the dynamic current source and/or further signal processing of SD data will reduce the affects of this type of uncorrelated noise.

Due to potential unit-to-unit variations in the high frequency structural dynamics of the M6, an empirical analysis of a small group of accelerometers was used to develop the SD functions. The empirical analysis correlates specific diagnostic signatures to temperature and modes of failure. Figures 3-5 are graphic illustration from previous work of typical SD data related to specific soft failures.

The M6 accelerometer is a shear-mode design. It is stud mounted to a base pad using $40 \mathrm{in}-\mathrm{lb}$ torque. In a voltage-mode operation, both the piezoelectric charge coefficient and capacitance will affect output sensitivity. The sensor's fundamental "mounted" resonance is typically $40 \mathrm{kHz}$ at room temperature. Initial testing of the M6 accelerometer identified other resonant frequencies both for mounted and free-free conditions. Results from impact testing and using the reverse piezoelectric effect show the correlation between the sensor's resonance and the resonant/anti-resonant pairs of the SD spectral signature. A "free-free" boundary condition for the M6 accelerometer represents sensor separation from the base with cable attachment. Placing the sensor on a foam pad simulates this condition. As shown in Figure 6, the "free-free" resonances of the M6 accelerometer appear at 32,57 and $69 \mathrm{kHz}$. using both the forward and reverse piezoelectric effect.

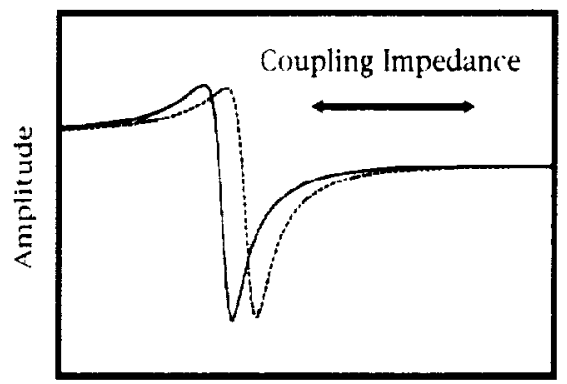

Frequency

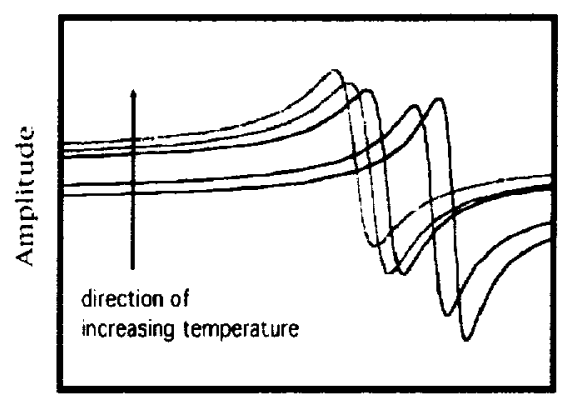

Frequency

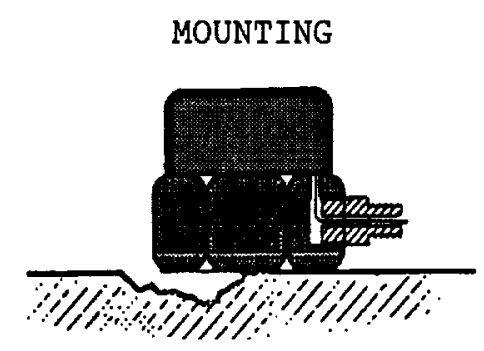

Figure 3: The SD signature of a compression-mode accelerometer as a function of mounting condition, i.e. loose, tight..

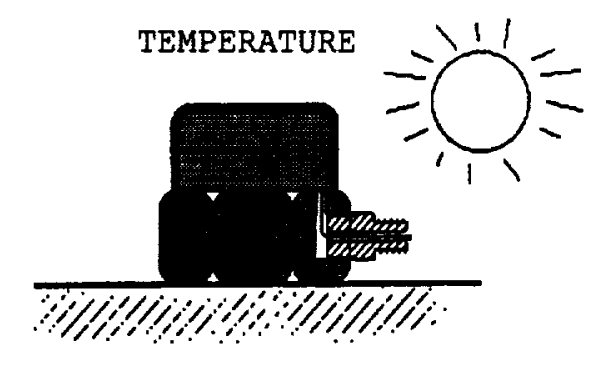

Figure 4: The SD signature of a compressionmode accelerometer as a function of ambient temperature. 

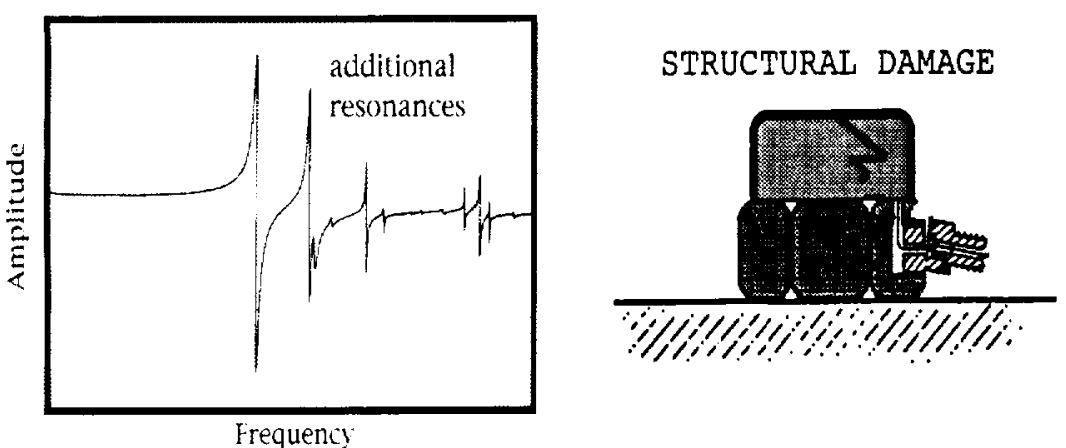

Figure 5: The SD

signature of a compression-mode accelerometer as a function of structural damage.
The location of the mounted and "freefree" resonance are sufficiently different to initially focus on the $30-100 \mathrm{kHz}$ range for $\mathrm{SD}$ function analysis and formulation. Differentiating the affects of temperature, mounting torque levels, and changes in base impedance, while minimizing the cross-sensitivity of the SD function/failure detection algorithm, is the goal of the test program. The SD function test program includes characterizing the diagnostic signatures produced by the M6 accelerometer due to changes in mounting torque, base impedance and temperature.

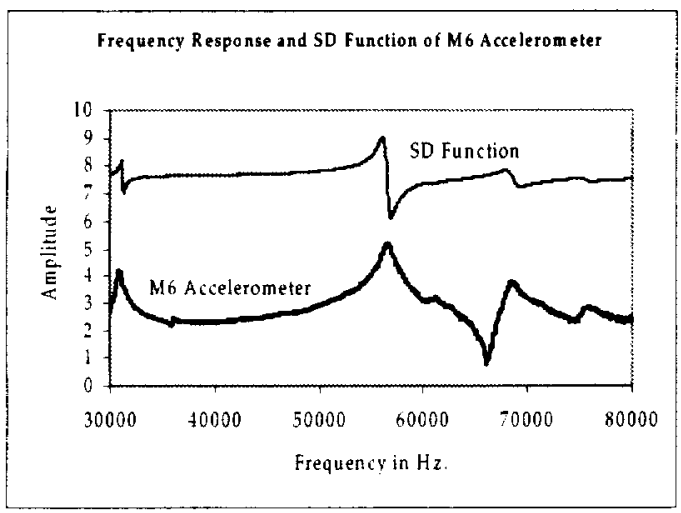

Figure 6: Impact testing of the M6 accelerometer using a "free-free" boundary condition produces a frequency response identifying the sensor's resonant frequencies. The resonant/anti-resonant pairs of the M6 accelerometer are also shown and correlate to accelerometer resonance.

\section{SD Function Test Program}

A computer-aided test system employed in earlier studies (ref. 1-6) was used to measure M6 diagnostic signatures. This system uses a two-channel digital signal analyzer to calculate the frequency response between input electrical drive and output voltage across a fixed load capacitance. Data from the frequency response function is processed and correlated to soft and hard failures. Potential SD functions are developed from these test results.

In the second phase, algorithms for the $\mathrm{SD}$ functions are downloaded to a prototype Self-Diagnostic M6 Accelerometer System and tested. The prototype Self-Diagnostic M6 Accelerometer System has a programmable frequency band using a periodic chirp drive and calculates, in-situ, diagnostic conditions. The dynamic current source includes automatic gain control in addition to software control of both bandwidth and resolution of the $\mathrm{SD}$ test frequencies.

The prototype M6 accelerometer system hardware includes a "T" connector box interfaced to a data acquisition and control unit. The M6 accelerometer is connected to the "T" box using a low noise, low capacitance coaxial cable. A BNC signal jack can be used to monitor output acceleration and the unprocessed SD signature. The data acquisition and control unit measures and collects the SD signatures from the " $\mathrm{T}$ " box. A five-line 
LCD display allows "real-time" viewing of information concerning M6 operation. Diagnostic data is transferable to a computer using a serial port. Program modification and upgrading are also permitted using a second serial port. Tests conducted with the prototype unit helped verify the operation of the SD functions

\section{Identifying SD Functions}

\section{Affects of Base Impedance on the M6}

Accelerometer

As predicted by the reverse piezoelectric effect transfer function, base mounting impedance will change the number and location of the resonant/anti-resonant pairs. Figure 7 illustrates the change in SD signature due to the shape and material properties of the mounting block. The dynamic range of the resonant/anti-resonant pairs can also fluctuate because of the constructive or destructive influence of the base eigenvalues on the SD signature. The "loose" SD signatures for each base condition are similar approaching the signature for the "free-free" boundary condition. The complexity of the SD signature increases above $70 \mathrm{kHz}$. At least one discrete resonant/antiresonant pair exists between 37 and 65 $\mathrm{kHz}$ for all three base impedances.

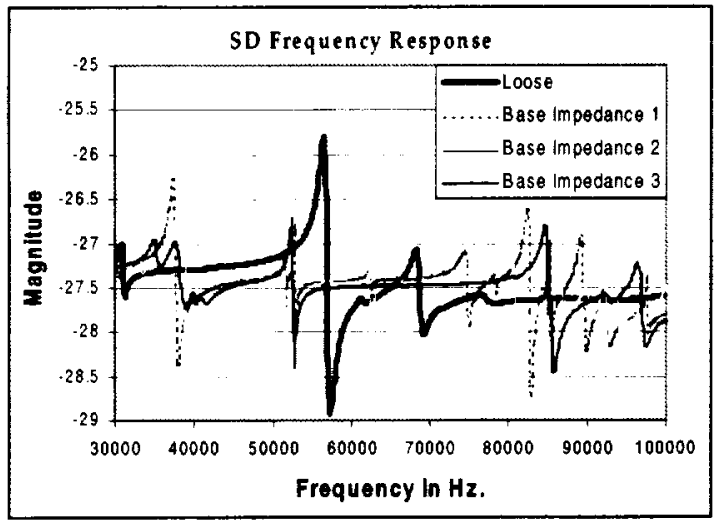

Figure 7: Affects of base impedance on SD data. SD data of the "loose" M6 is common to all three base impedances
Affects of Mounting Torque on the M6 Accelerometer

Changing mounting torques from 25 to 40 in-lbs measurably shifts the spectral resonance of the diagnostic signature. As illustrated in Figure 8, a resonant/antiresonant pair can shift as much as $1000 \mathrm{~Hz}$ as mounting torque increases. This magnitude of frequency shift is not observed for all resonant/anti-resonant pairs and can be affected by the base impedance. However, for the three base impedances tested, as mounting torque increases, the resonant/anti-resonant pairs continue to shift to higher frequencies until the M6 is properly mounted. Once the M6 is loose (but still threaded to the base), the mounted SD signature shifts dramatically as illustrated in Figure 7.

Based on repeatability tests, a 5 in-lb mounting torque resolution can be measured using SD data. Further results show that the resolution of the torque measurement using the spectral shift of the SD signature usually degrades at frequencies above $70 \mathrm{kHz}$. Minor changes in base mounting impedance including adding a washer can significantly change the SD signature. For example, a washer between the M6 and base impedance shifts the resonant/anti-resonant pairs to lower frequencies and changes the dynamic range of the resonant/anti-resonant pairs.

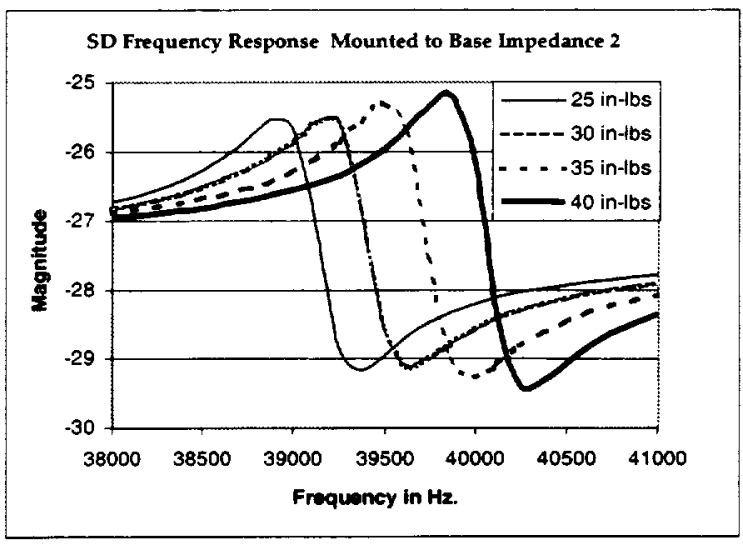

Figure 8: SD data of the M6 accelerometer mounted to base impedance 2 using 25. 30 . 35 and 40 in-lb torque levels. 
Affects of Temperature on the M6 Accelerometer

To help simulate the temperature conditions of the shuttle pumps in operation, both liquid nitrogen and an environmental control chamber are used. Cold temperature testing involves quenching the sensor in a liquid nitrogen bath. The SD spectral signature is measured as the M6 cools. After the SD signature stabilizes, the M6 is removed from the liquid nitrogen bath and diagnostic signatures are recorded until the M6 reaches room temperature. No temperature sensor is mounted to the M6 since this will load the sensor's housing thereby affecting the assembly impedance and diagnostic signatures. As shown in Figure 9 , the bias level of the SD signature and the location of the resonant/antiresonant pairs continuously shift as the M6 accelerometer warms from cryogenic to room temperature. Condensation in the form of frost developed on the sensor. This event did not appear to significantly affect the bias level data but, in some cases, damped the resonant/anti-resonant pairs. Little change in the damping characteristics of the resonant/anti-resonant pairs was recorded for the "loose" M6 when exposed to this event. The bias level. and spectral location and shape of the resonant/antiresonant pairs for the diagnostic signature returns to the original state once ambient conditions are restored.

High temperature testing involved placing the M6 with mounting base inside a Despatch environmental chamber. A controlled temperature ranging from $23^{\circ} \mathrm{C}$ $\left(73^{\circ} \mathrm{F}\right)$ to $90^{\circ} \mathrm{C}\left(194^{\circ} \mathrm{F}\right)$ was maintained during this study. The high temperature diagnostic signatures, in Figure 10, also show a shift in resonance frequency and bias with temperature. The resonant frequencies shift with increasing temperature in the same direction as the low temperature test, while the bias shifts in the opposite direction. Monitoring temperature requires tracking both the $\mathrm{SD}$ signature bias level and the location of the resonant/anti-resonant pairs.

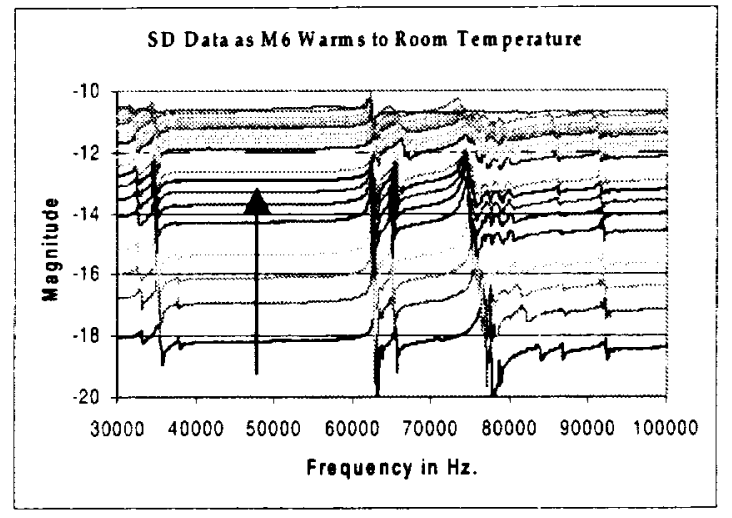

Figure 9: SD data of the mounted (base impedance 3) M6 accelerometer as it warms from cryogenic to room temperature. The arrow shows direction of SD signature bias level as the M6 warms.

Affects of the Electrical Drive Signal on the M6 Accelerometer

The M6 accelerometer was tested to determine the changes in SD signature due to a $20 \mathrm{~dB}$ change in electrical drive level. This study measures the affect of automatic gain control for the electrical drive. A minute difference in the SD function due to the $20 \mathrm{~dB}$ increase occurs at the resonant/anti-resonant pairs. Long term test results comparing different drive levels within the $20 \mathrm{~dB}$ range show no significant change that would affect the development of the SD functions.

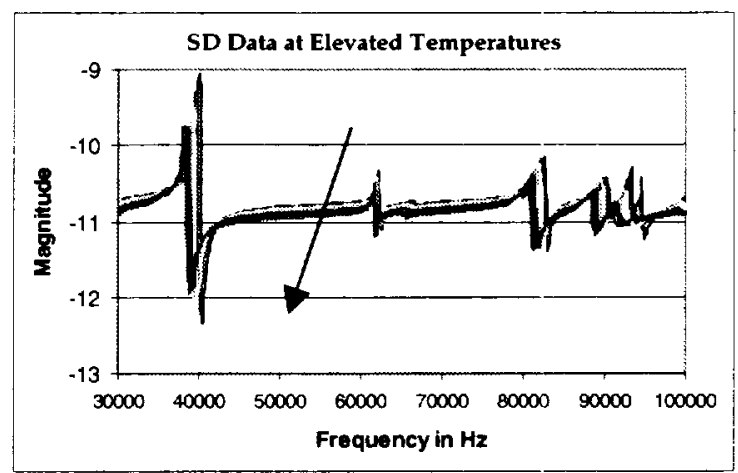

Figure 10: SD data of the mounted (base impedance 2) M6 accelerometer at elevated temperatures. The angled arrow shows direction of SD signature bias level and frequency shift due to increased temperature from 23 to $90 \mathrm{C}$. 


\section{SD Functions and the Prototype System}

Three SD functions are proposed to identify and/or measure (1) a "loose" M6 mounting condition (2) a correctly mounted M6 accelerometer and (3) environmental temperature. Since base mounting impedance can alter the location and dynamic range of the resonant/antiresonant pairs, a self-calibration for the mounted M6 is required for each new base. The "loose" SD signature is not affected by the base impedance and therefore is a standardized signature for each M6 accelerometer. A test of the "loose" M6 SD signature checks the piezoelectro-mechanical properties before torque-down. The "loose" SD function for each M6 is archived in the SD system.

The SD signature for the correctly mounted M6 is recorded immediately after installation. A broadband sweep of the diagnostic frequency band identifies the location and dynamic range of each resonant/anti-resonant pair for the mounted M6. The resonant/anti-resonant pair with the peak dynamic range centers the SD frequency band. The frequency range of the SD signature extends to include the primary "loose" resonant/ anti-resonant pair at $57 \mathrm{kHz}$. Potential variations in environmental temperature and sensitivity to mounting torque typically require further expansion of the frequency range by as much as $5000 \mathrm{~Hz}$, adding $2500 \mathrm{~Hz}$ to the lower and high ends of the frequency range. Base impedance tests show that several discrete resonant/anti-resonant pairs for the mounted M6 appear in the 35 to $65 \mathrm{kHz}$ range. All are good candidates for diagnostic detection. This band also includes the "loose" resonant/antiresonant pair at $57 \mathrm{kHz}$. A step-by-step process for installing the M6 using the SD system is shown in Figure 11.

The installation process for the M6 accelerometer begins by measuring, insitu, the "loose" SD signature. This is a broadband SD signatures exciting the 30 to $80 \mathrm{kHz}$ frequency range. Measured SD data is analyzed to determine bias level and position of the resonant/anti-resonant frequencies. The acceptance criterion compares the measured bias level and resonant/anti-resonant frequencies to the SD function data for the "loose" M6 mount condition. If acceptable, the temperature data is recorded and SD function sensitivity to torque level is checked next. The torque sensitivity test requires remounting the M6 using several different torque levels. A broadband, high resolution SD signature is recorded for each torque setting. This test identifies frequency band sensitivity to changing torque levels. The torque sensitive frequency range is included in the operational SD frequency band. Following this test, the M6 is properly mounted to the base pad. The broadband, highresolution $\mathrm{SD}$ signature is again measured and temperature level is calculated using the SD temperature function. This temperature reading is compared to the "loose" mount temperature measurement as a check. The operational or "tuned" SD frequency band is set based on specific data including the torque sensitivity test results, the dynamic range of the resonant/anti-resonant peaks and the "loose" SD signature. Comparing temperature calculations using both broadband and tuned SD signatures confirms the operation of the SD temperature function using a narrow tuned SD frequency band. If calculated temperature of tuned SD frequency band differs significantly from the broadband measurement, the tuned SD frequency band is expanded and retested.

Incremental expansion continues until the temperature reading agrees or reaches the broadband SD frequency band. The frequency band is kept as small as possible to minimize diagnostic cycle time.

At this time, the prototype system has been programmed to test to following:

1) Operation of the broadband, high resolution SD signature to detect resonant/anti-resonant peaks of the mounted and loose M6 accelerometer. 


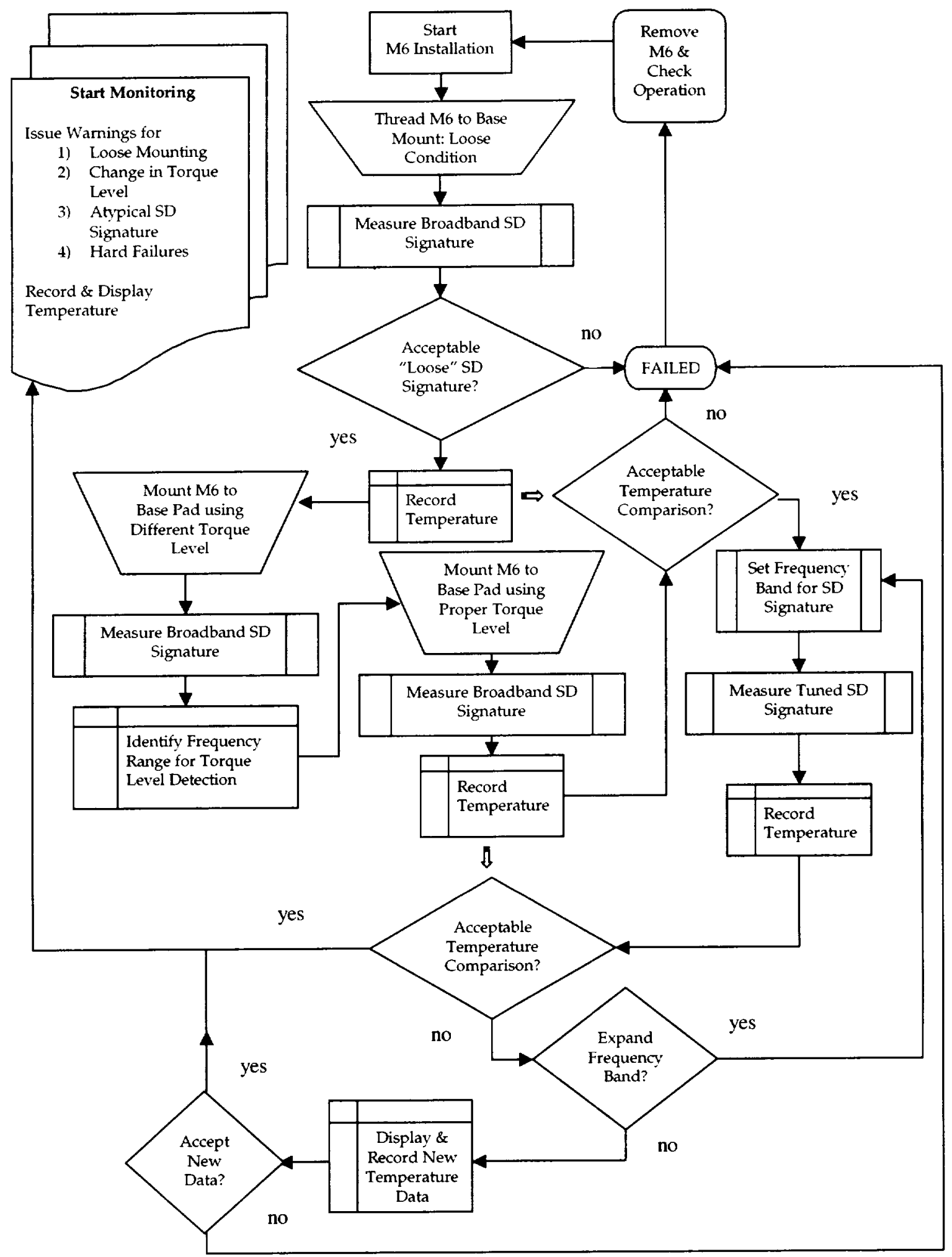

Figure 11: Flow chart of SD system procedure for installing M6 accelerometer. 
2) Procedure to set the tuned frequency range for the SD signature.

3) Performance of the operational SD signature to detect "loose" and "mounted" conditions

4) Performance of the SD temperature function versus temperature.

5) Performance of the hard failure detection mode when the termination impedance of the sensor abruptly changes to either an open or short circuit condition.

All four functions have been successfully tested using an estimated one-second diagnostic cycle time. Additional software procedures outlined in Figure 11 are being added to the SD system.

\section{Conclusions}

This paper discusses the general operation of the M6 accelerometer SD system and procedures for developing and evaluating the SD system. Results show that analyzing the high frequency spectral characteristics of the M6 accelerometer's reverse piezoelectric effect can significantly enhance the diagnostic capabilities of the sensor. Specific hard and soft failures exhibit unique signatures that can be detected using mathematical (SD) functions while compensating for environmental temperature. This highspeed self-diagnostic sensor is especially important in complex propulsion systems.

\section{References}

1) Atherton, W. J. and Flanagan, P. M., "A Self Diagnostic System for Piezoelectric Sensors ", AIAA paper no. 89-2638,

AIAA/ASME/SAE/ASEE 25th Joint Propulsion Conference, Monterey, Ca., July 10-12, 1989

2) Flanagan, P. M and Atherton, W. J., "Developing a Self-Diagnostic System for Piezoelectric Sensors", AIAA paper no. 90-2230, AIAA/ASME/SAE/ASEE 26th Joint Propulsion Conference, Orlando, Fl., July 16-18, 1990

3) Flanagan, P. M, "In-Situ

Measurement of Sensitivity for a Piezoelectric Sensor", AIAA paper no. 91-2521,

AIAA/ASME/SAE/ASEE 27th Joint Propulsion Conference, Sacramento, Ca., June 24-26, 1991

4) Flanagan, P. M, "Design of a SelfDiagnostic Beam-Mode Piezoelectric Accelerometer", AIAA paper no. 923656, AIAA/ASME/SAE/ASEE 28th Joint Propulsion Conference, Nashville, Tenn., July 6-8, 1992

5) Flanagan, P. M, "Dedicated System for a Self-Diagnostic Piezoelectric Accelerometer", AIAA paper no. 942982, AIAA/ASME/SAE/ASEE 30th Joint Propulsion Conference, Indianapolis, In., June 27-29, 1994

6) Flanagan, P. M, "Design of a SelfCompensation System for Piezoelectric Accelerometers", AIAA paper no. 962935, AIAA/ASME/SAE/ASEE 32th Joint Propulsion Conference, Lake Buena Vista, Fl., July 1-3, 1996 


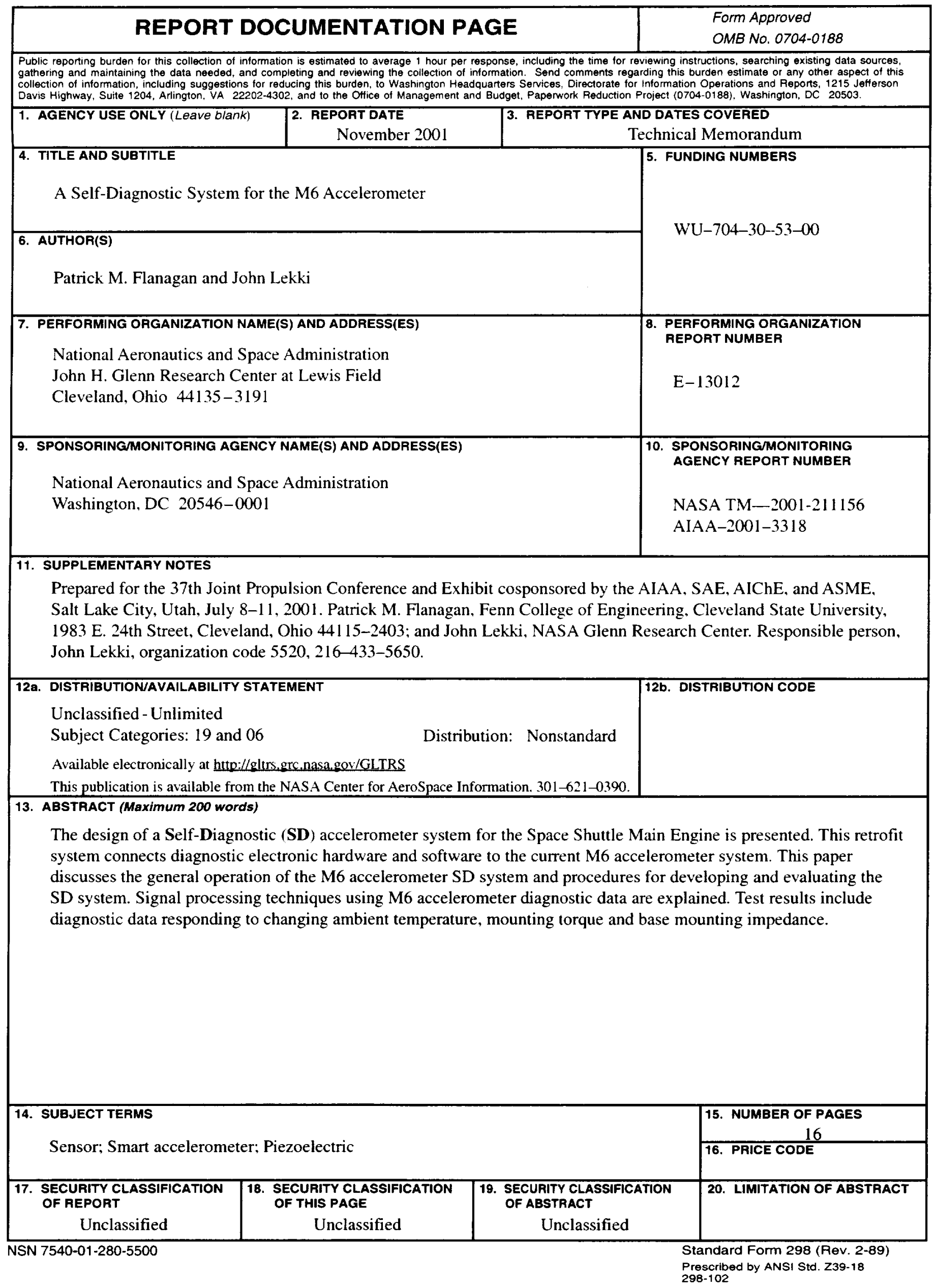


